\title{
A Case of Bilateral Calcaneal Fracture, Following Fall from 35 Feet
}

\section{Purushothama Sastry, Sujana Theja JS, Supreeth N and Arjun Markanday*}

Department of Orthopaedics, JSS Hospital, Mysore, India

\begin{abstract}
Introduction: Called Lover's Fractures, the calcaneus commonly fractures due to fall from height. The calcaneus is the most frequently fractured tarsal bone. Tarsal bone fractures account for about $2 \%$ of all adult fractures. Of these, $60 \%$ are calcaneus fractures. The heel bone is often injured in a high-energy collision where other parts of the skeleton are also injured. In up to $10 \%$ of cases, the patient can also sustain a fracture of the spine, hip, or the other calcaneus. Injuries to the calcaneus often damage the subtalar joint and cause the joint to become stiff. This makes it difficult to walk on uneven ground or slanted surfaces.

Case Report: A 24 year old male, working as a lift operator presented to the casualty of the hospital after the elevator broke down and came down in a free fall from about a height of 4 stories (35 feet). He presented along with the other occupants of the lift who also sustained calcaneal fractures. The case on arrival was subjected to ATLS protocol and through radiologic work up was done. Following a period of 12 days post trauma he was operated for bilateral calcaneal fractures and discharged 10 days post operatively.

Conclusion: Calcaneal fractures continue to tread the fine line between operative and conservative management. In a young individual an operative management is likely to give a better outcome as the associated soft tissue problems, commonly encountered, yield a better outcome and faster healing rates. Used of closed reduction further helps alleviate the need to worry about wound healing
\end{abstract}

Keywords: Calcaneal fracture; Canulated cancellous screws; Fracture blister

\section{Introduction}

Epidemiology

Incidence

1. Most frequent tarsal fracture

2. $17 \%$ open fractures

\section{Pathophysiology}

\section{Mechanism}

1. Traumatic axial loading is the primary mechanism of injury

2. fall from height

3. motor-vehicle accidents

\section{Pathoanatomy}

Intra-articular fractures

- Associated injuries

○ Orthopaedic

- extension into the calcaneo-cuboid joint occurs in $63 \%$

- vertebral injuries in $10 \%$

- contralateral calcaneus in $10 \%$

- Prognosis

○ Poor with $40 \%$ complication rate

- Increased due to mechanism (fall from height), smoking, and early surgery

- lateral soft tissue trauma increases the rate of complication

The optimal management of intra-articular calcaneal fractures is controversial. The goal of operative management is to achieve anatomic joint reduction, and restore height, length, width and axis of the calcaneus. Stable internal fixation should allow early motion to restore function. Open reduction and stable internal fixation is usually advocated for intra-articular fractures with relevant joint displacement $(.1 \mathrm{~mm})$, and in extra-articular fractures compromising the soft tissues and/or with unacceptable positioning, shortening and malalignment of the calcaneus (.108 valgus/.58 varus). Relative contraindications include old age, significant co-morbidities, smoking, diabetes mellitus, use of steroid medications and vascular insufficiency. Several large series of intra-articular fractures that were classified by CT scanning and managed with open reduction and internal fixation (ORIF) showed good to excellent results in $60-85 \%$ of cases. However, correlation between anatomical restoration and outcome (function, quality of life) has not been proven unambiguously, while calcaneal fractures are notorious for post-operative complications. Injury to the soft-tissue envelope of the hindfoot affects both the timing of surgery and wound healing. Wound healing problems occur in $16-25 \%$ of patients after ORIF of calcaneal fractures, and have been reported to be as high as $43 \%$. Other forms of operative management are external fixation minimally invasive percutaneous fixation and arthroscopically assisted fixation. Non-operative management consists of elevation, ice, analgesia and early ankle and subtalar joint mobilization. Some clinicians advocate the use of a splint for 2 weeks to prevent equinus of the ankle and allow soft tissue healing. Weight bearing is initially restricted to prevent further collapse of the fracture. Impulse compression application

*Corresponding author: Arjun Markanday, Department of Orthopaedics, JSS Hospital, Mysore, India, Tel: 0821233 5555; E-mail: arjunmarkanday@yahoo.com

Received April 14, 2015; Accepted May 26, 2015; Published June 04, 2015

Citation: Sastry P, Theja JSS, Supreeth N, Markanday A (2015) A case of Bilateral Calcaneal Fracture, Following Fall from 35 Feet. J Trauma Treat 4 254. doi:10.4172/2167-1222.1000254

Copyright: ( 2015 Sastry P, et al. This is an open-access article distributed under the terms of the Creative Commons Attribution License, which permits unrestricted use, distribution, and reproduction in any medium, provided the original author and source are credited. 
has been proposed as a possible intervention to reduce swelling and improve range of motion and function. Although the advantages of operative versus non-operative management have been questioned, many orthopaedic surgeons approach displaced calcaneal fractures operatively.

\section{Case Report}

- A 24-year-old male presented to the hospital casualty after the elevator he was in descended 4 floors (35 feet) after a malfunction. The impact caused him to present with swelling and exquisite tenderness of both feet and ankles.

- Immediately on arrival the ATLS protocol was followed and radiologic work up done.

- He developed fracture blisters fourth day post admission which were promptly drained and dressing done (Figures 1 and 2).

- After waiting for 12 days post trauma he was taken up for surgery once the blisters had healed (Figure 3).

\section{General Examination}

A moderately built male with all vital parameters with in normal limits.

\section{Local Examination}

Tense swelling around both the ankles and the heels.

Range of motion restricted at the ankle.

Other joints in the body including the spine did not reveal any abnormality on examination.

\section{Investigations}

Laboratory Investigations:

Hb: 14.2 gm\%

Immune status: Negative for HIV, $\mathrm{HBsAg}$ and $\mathrm{HCV}$

Other hematological investigations were normal.

X-Ray: Bilateral calcaneal fracture

CT Scan (Right): comminuted displace fracture of the calcaneum with articular extension into the subtalar joint and surrounding soft tissue edema (Figure 4).

\section{Operative Procedure}

Under C-arm guidance the patient was placed in a prone position and tourniquet applied.

The right side calcaneus was operated on first. A thick K-wire was inserted into the tuberosity and used a joystick to move the fragments. Guide wires were placed after obtaining a satisfactory reduction on both lateral and axial views. This reduction was appreciated under direct vision and provisionally fixed with $2.5 \mathrm{~mm}$ K-wires passed axially. On table a radiographic assessment was made for restoration of Bohlers and Gissane's angle. On achieving acceptable reduction, especially of posterior facet, final fixation was done with 3 Canulated Cancellous Screws.

3 Canulated Cancellous Screws (CCS) were used to fix the calcaneum on the Right side.

Next the Left Calcaneus was operated under a similar fashion of

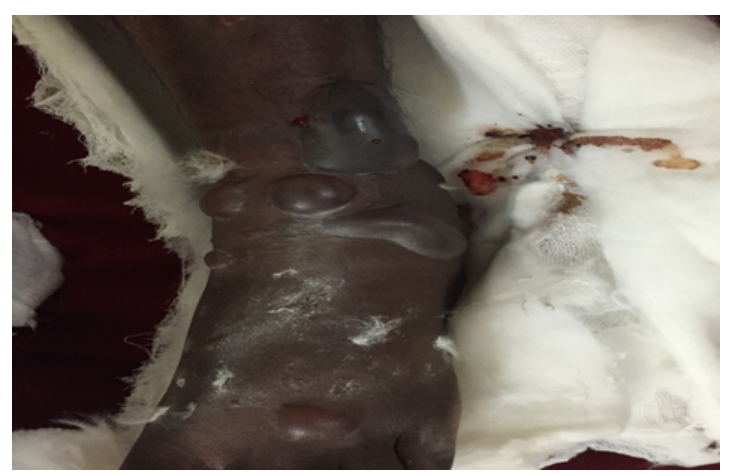

Figure 1: Fracture blister on day 4 post trauma.

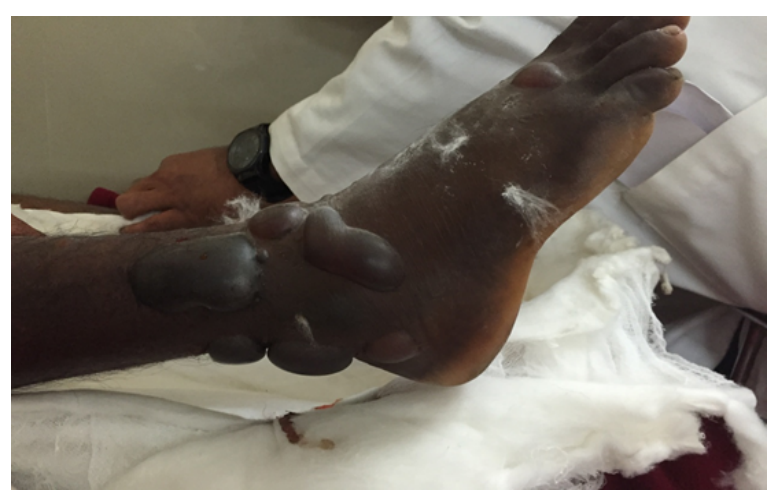

Figure 2: Lateral view of the blisters.

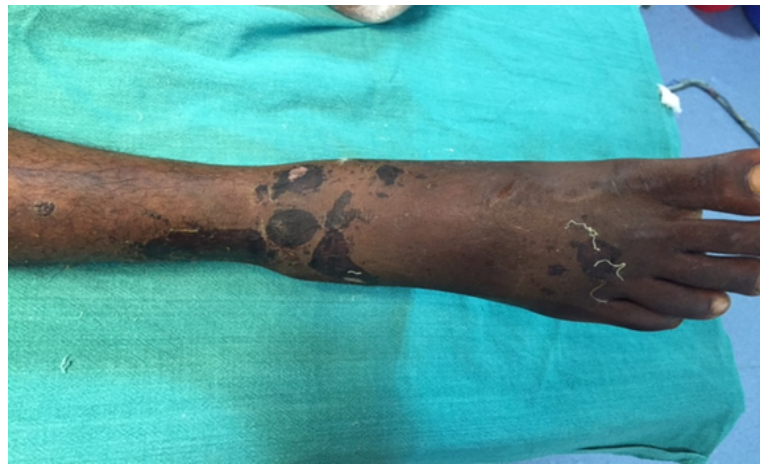

Figure 3: Day 12 post trauma (blisters subside).

using a K-wire as a joystick and a single CCS was used to fix the fracture after getting good purchase up to the sustentaculum tali.

Both the reductions were checked under $\mathrm{C}$-arm and below knee POP slabs were applied to both the legs.

\section{Discussion}

Intra-articular fractures are common as compared to extraarticular fractures, hence the importance of anatomical reduction to decrease the possibility of joint incongruity and subtalar arthritis. Open reduction and internal fixation is now more aggressively advocated for management of intra-articular fractures of calcaneum. Intra-articular fractures are commoner and hence importance of anatomical reduction 


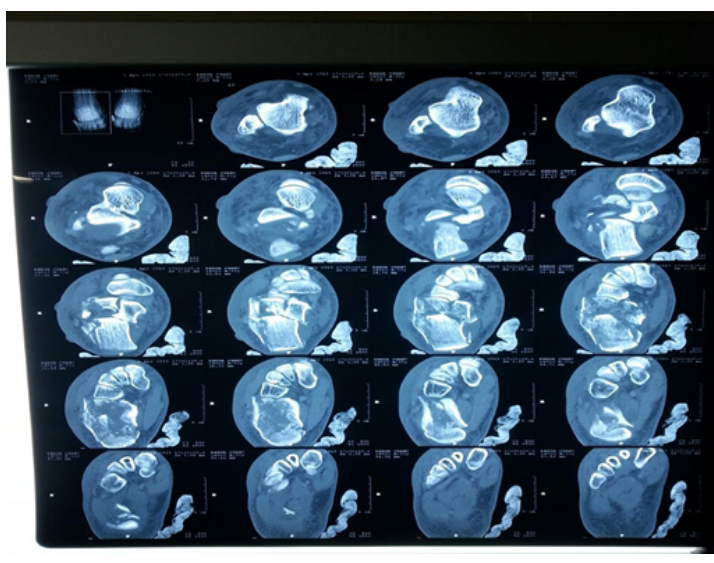

Figure 4: CT scan of the Right calcaneum.

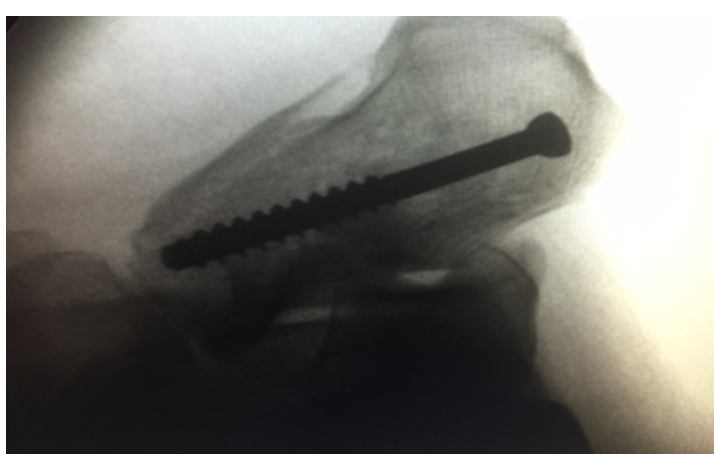

Figure 5: Intra op reduction of Left calcaneum.

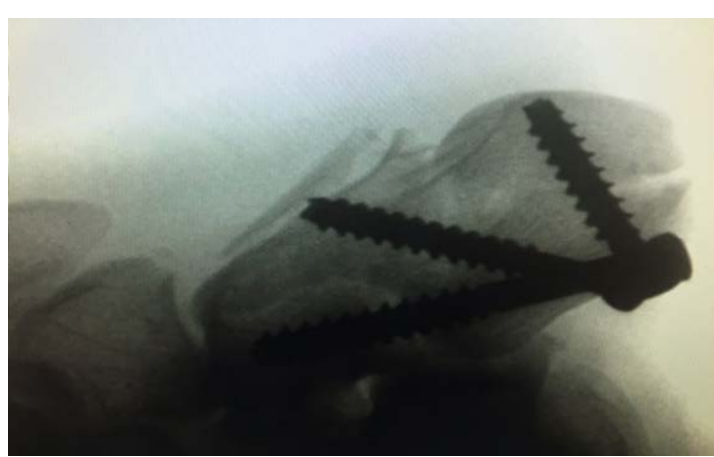

Figure 6: Intra op reduction of Right Calcaneum.

to decrease the complication of subtalar arthritis, joint incongruity and late ankle arthritis.

Restoration of the mechanical stability for earlier full weightbearing and patient mobilization with minimal morbidity and complications, were the surgical treatment goals. The principal aims of surgical treatment include restoration of height, length, width, and axis with anatomical reconstruction of all joint surfaces and restoration of function by primary stable osteosynthesis. Options include open reduction combined with internal fixation (ORIF) with or without grafting or bone cement augmentation, wire circular frame procedure, open reduction and arthroscopically assisted internal fixation and primary subtalar arthrodesis. Soft tissue protection is important in surgical management of the calcaneus (Figures 5 and 6). Folk et al. [1-4] in their study including operative treatment of 190 calcaneal fractures, showed that in $25 \%$ of cases there was some form of wound complication and $21 \%$ of these required surgical treatment.

However for the prevention of soft tissue problems and known delays in healing the Closed reduction and internal fixation with canulated cancellous screws provide a better method to stabilize the calcaneus.

\section{Conclusion}

Calcaneal fractures continue to tread the fine line between operative and conservative management. In a young individual an operative management is likely to give a better outcome as the associated soft tissue problems, commonly encountered, yield a better outcome and faster healing rates.

\section{References}

1. Fitzgibbons TC, McMullen ST, Mormino MA (2001) Fractures and dislocations of the calcaneus. In: Bucholz RW, Heckman JD (eds). Rockwood and Green's Fractures in adults, Vol.3, 5th ed. Philadelphia: Lippincott Williams \& Wilkins, 2133-2179.

2. Murphy GA (2003) Fractures and dislocations of foot. In: Canale ST (ed.) Campbell's operative Orthopaedics, Vol.4, 10th ed. Philadelphia: Mosby Inc., 4231-4283.

3. Kundel K, Funk E, Brutscher M, Bickel R (1996) Calcaneal fractures: operative versus nonoperative treatment. J Trauma 41: 839-845.

4. Cave EF (1963) Fracture of the os calcis--the problem in general. Clin Orthop Relat Res 30: 64-66. 